TITLE:

\title{
Formalism to model stacking fault effects on surface phase stability in alloys
}

$\operatorname{AUTHOR}(\mathrm{S})$ :

Yuge, Koretaka; Saito, Ryuichiro; Kawai, Jun

\section{CITATION:}

Yuge, Koretaka ...[et al]. Formalism to model stacking fault effects on surface phase stability in alloys. Physical Review B 2013, 87(2): 024105.

ISSUE DATE:

2013-01-09

URL:

http://hdl.handle.net/2433/188003

RIGHT:

(C)2013 American Physical Society 
PHYSICAL REVIEW B 87, 024105 (2013)

\title{
Formalism to model stacking fault effects on surface phase stability in alloys
}

\author{
Koretaka Yuge, Ryuichiro Saito, and Jun Kawai \\ Department of Materials Science and Engineering, Kyoto University, Sakyo, Kyoto 606-8501, Japan
}

(Received 1 August 2012; published 9 January 2013)

\begin{abstract}
Based on the first-principles calculation, we propose a formalism to model stacking fault effects on configurational energetics at the surface, which can be additional major factors governing surface phase stability in alloys with the example of the close-packed surface of Pt-Re alloys. The Pt-Re alloys exhibit several stable surface structures with stacking sequences different from the bulk for ground as well as random states. Stacking sequences for stable surfaces significantly depend on surface composition, which is mainly due to the marked coupling contribution between stacking and atomic configurations to the total energy. The proposed formalism successfully confirms the importance of stacking fault effects on surface phase stability in alloys.
\end{abstract}

DOI: 10.1103/PhysRevB.87.024105

PACS number(s): 68.35.bd, 81.30.-t

\section{INTRODUCTION}

Recent progress in computational techniques has enabled us to accurately predict a variety of properties of bulk alloys based on density functional theory (DFT); however, the situation becomes rather complicated for surfaces, since atomic configuration and composition at the surface can differ from those in bulk due to surface segregation. Surface segregation was first proposed by Gibbs at the end of the nineteenth century, and a surface sensitive experiment first investigated this phenomenon around three decades ago. ${ }^{1,2}$ Today, surface phase stability, including the behavior of surface segregation and structure on an atomic scale, has become fundamentally and technologically important, and has been actively investigated by a substantial amount of experimental work since the resultant surface-specific structures can exhibit outstanding characteristics, such as catalytic and corrosion properties that cannot be achieved by the bulk. ${ }^{3-9}$ So far, surface phase stability has also been theoretically well investigated based on DFT, and is believed to be well described in terms of surface orientation, competition between atomic ordering tendency and segregation, molecular adsorption, and bulk composition. ${ }^{10-16}$

In this paper, we confirm another important factor, stacking faults at the surface, which can make a major contribution to describing surface phase stability. Surface structure is generally described by the stacking of constituent planes parallel to the surface and, geometrically, there can be stacking sequences at the surface that are different from those of the bulk, particularly for alloys. One of the most representative examples is the close-packed surface of fcc-hcp binary alloys, since fcc and hcp can be described by the similar stacking sequences of $\mathrm{ABCABC} \cdots$ and $\mathrm{ABABAB} \cdots$, respectively. ${ }^{17}$ At the surface, there is naturally a great possibility that surface structures with stacking sequences different from bulk (e.g., mixed stacking of fcc and hcp in this case) will become ground states when surface segregation takes place. Stacking faults at the surface have been commonly discussed for atomic adsorption on the substrate surface. ${ }^{18,19}$ Although the stacking fault effects on surface phase stability in configurational energetics has been considered to play an important role, it has not been quantitatively confirmed so far since they might have complicated coupling effects between atomic configuration and stacking sequences on total energy at the surface. In order to demonstrate the effects of stacking faults, we investigated phase stability for the close-packed surface of fcc-hcp, Pt-Re binary alloys, based on first-principles calculation with DFT. We will show that for ground states as well as random states, surface structures with stacking faults can be more stable than those with bulklike stacking, indicating that the inclusion of stacking fault effects is fundamental for accurate description of surface phase stability.

\section{METHODOLOGY}

In order to quantitatively describe how stacking faults affect surface phase stability, we should know the dependence of total energy for the surface in terms of possible atomic configuration, composition, and stacking sequences. This requires significant computational costs, which are typically far beyond the practical application of first-principles calculations. So our strategy was to combine the first-principles calculation with variable-lattice cluster expansion (VLCE) ${ }^{20,21}$ developed by the authors, which provides effective Hamiltonian including the contribution from atomic configuration, composition, and stacking sequences, which overcomes the above practical difficulty. Within the VLCE ansatz, the configurational energy $E$ at the surface is given by Fig. $1{ }^{20}$ Here, expansion coefficients $V \mathrm{~s}$ are called effective cluster interactions (ECIs), $\sigma_{i}$ denotes the pseudospin variable to specify the occupation of lattice site $i\left[\sigma_{i}=+1(-1)\right.$ denotes $\mathrm{Pt}(\mathrm{Re})$ occupation], $\tau_{\Lambda}$ denotes the pseudospin variable to specify stacking sequences for surface layer $\Lambda\left(\tau_{\Lambda}=0,+1,-1\right.$ denote $\mathrm{A}, \mathrm{B}, \mathrm{C}$ stacking $)$, and $\rho$ s are functions of $\tau_{\Lambda}$. Open circles denote base lattice points that can be occupied by constituent elements, and open squares denote virtual lattice points specifying stacking sequences. $\alpha$ and $\beta$ represent clusters consisting of lattice points on base and virtual lattices, respectively. In the present system, we have two basis functions of $\rho$ s for the virtual lattice point: $\rho_{1}=\sqrt{3 / 2} \tau$ and $\rho_{2}=-\sqrt{2}\left(1-3 \tau^{2} / 2\right){ }^{20}(L)$ in Fig. 1 specifies the set of index of the basis function $\rho$ (i.e., 1 or 2): For instance, a pair cluster on the virtual lattice has $(L)$ of $(1,1),(1,2),(2,1)$, and $(2,2)$ representing the basis index on the constituent two lattice points. Summation in Fig. 1 is taken over all possible independent contributions to $E$, which is determined by the procedure in Ref. 21 . The first term on the right-hand side of Fig. 1 is independent of configuration as well 


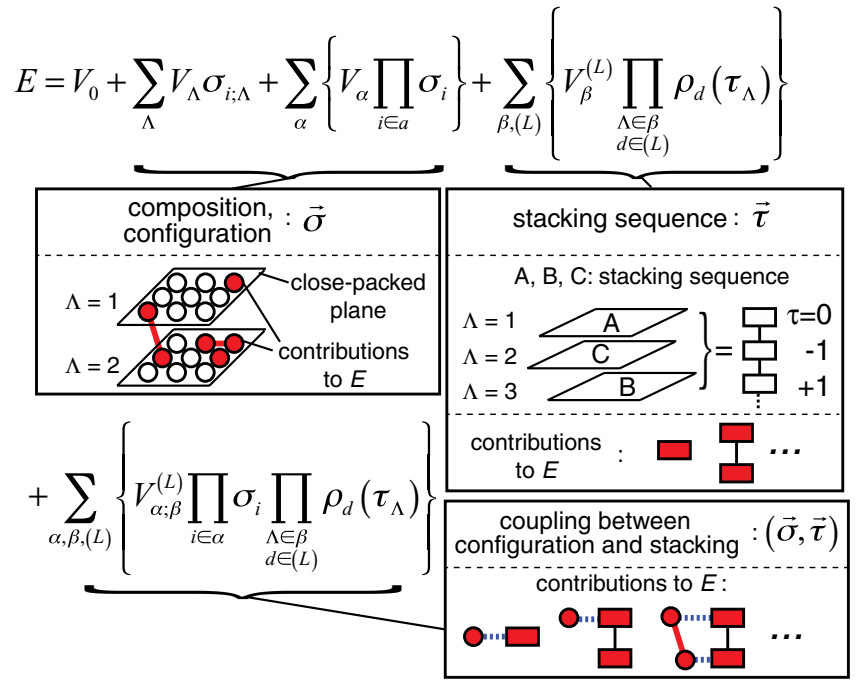

FIG. 1. (Color online) Proposed formalism and concept to treat possible composition, atomic configuration, and stacking sequences for configurational energetics at close-packed surfaces. Open circles denote lattice points that can be occupied by constituent elements, and open squares denote "virtual" lattice points to specify stacking sequence. Lattice points filled with red color represent contributions to total energy $E$ from each term in the equation.

as stackings. The second term corresponds to the contribution from the composition at each layer $\Lambda$, the third term from atomic configuration, the fourth term from stacking sequences, and the last term from coupling between atomic configuration and stacking. Inclusion of the last two terms is required to assess the stacking fault effects, which have been neglected in previous first-principles-based theoretical approaches. ${ }^{13}$ As schematically illustrated by open circles or squares filled with red color in Fig. 1, each contribution is described in terms of "clusters" composed of lattice points of close-packed plane and/or those of "virtual" lattice points: The former specifies atomic configuration and the latter specifies stacking sequences. The values of ECIs are determined from a set of first-principles calculations for surface slabs by employing least-squares fitting to Fig. 1. The number of surface structures is typically $\sim 100$ to assess surface phase stability in alloys. In the present system, however, a larger number of surface structures is required since we assess multiple stacking sequences simultaneously, while the previous approach is confined to single stacking. We calculate the total energy of 726 surface slabs with a variety of atomic configuration, composition, and stacking sequences. ${ }^{22}$ In the present study, we estimate the electronic contribution to the total energy, and the vibrational contribution is neglected: The inclusion of vibrational effects is out of our present scope. These structures are iteratively obtained by VLCE and first-principles calculations in order to accurately estimate ground states and random states at high temperatures: Randomly selected initial structures are used to obtain ECIs, then perform Monte Carlo (MC) simulation to obtain low- and high-energy surface structures different from the initial structures, which are added to obtain modified ECIs, and then perform the same procedure until the cross-validation $(\mathrm{CV})$ score,${ }^{23-25}$ which represents the predictive accuracy of the ECIs, is minimized by a genetic $\operatorname{algorism}^{26,27}$ and the energy for ground-state structures of VLCE becomes consistent with the DFT result. ${ }^{28,29}$ Total energies of the surface slabs are estimated by first-principles calculation using the VASP code $\mathrm{e}^{30,31}$ based on the projectoraugmented wave method ${ }^{32,33}$ within the generalized-gradient approximation of Perdew-Burke-Ernzerhof (GGA-PBE) ${ }^{34}$ to the exchange-correlation functional. The plane-wave cutoff of $400 \mathrm{eV}$ is used, and atomic positions are relaxed until the residual forces become less than $0.01 \mathrm{eV} / \AA$. The lattice parameter parallel to the surface is kept fixed at $2.80 \AA$ for $p(1 \times 1)$ unit cells of the surface in order to mimic the epitaxial strain from bulk, where $2.80 \AA$ is obtained through cluster expansion of volume for bulk $\mathrm{Pt}_{75} \mathrm{Re}_{25}$ alloy at $T \sim 2000 \mathrm{~K}$ in random states. ${ }^{35}$ The surface slabs have $17 \AA \AA$ vacuum thickness and 11 layers, where the top five layers are treated as the surface, and the slabs consist of up to 176 atoms (i.e., up to 16 atoms in each layer). We obtain 111 ECIs with a CV score of $0.1 \mathrm{meV} /$ atom, which gives sufficient accuracy.

\section{RESULTS AND DISCUSSION}

Before discussing surface phase stability, it is fundamentally important to investigate the magnitude of ECIs, which gives insight into the individual contribution to energy at the surface. Figure 2 shows the optimized ECIs from the second to fifth terms in Fig. 1, clarifying which contribution dominates the energy at the surface of Pt-Re alloys. Here, ECIs for composition are uniquely specified by $\Lambda$, those for configuration by $\alpha$, those for stacking by $\beta$ and $(L)$, and those for coupling between configuration and stacking by $\alpha, \beta$, and $(L)$ in Fig. 1, respectively.

It is clear that ECI for composition at the top layer (i.e., $\Lambda=1$ ) has the dominant contribution with a negative value, which is one order of magnitude larger than other ECIs. This indicates that $\mathrm{Pt}$ atoms are expected to significantly segregate to the topmost layer for the whole composition as well as various stacking sequences. The other ECIs on the left- and right-hand sides in Fig. 2 are in a similar magnitude, and ECIs for coupling between configuration and stacking have the largest number. Therefore, our theoretical approach based on Fig. 1 reveals that surface phase stability of Pt-Re alloys

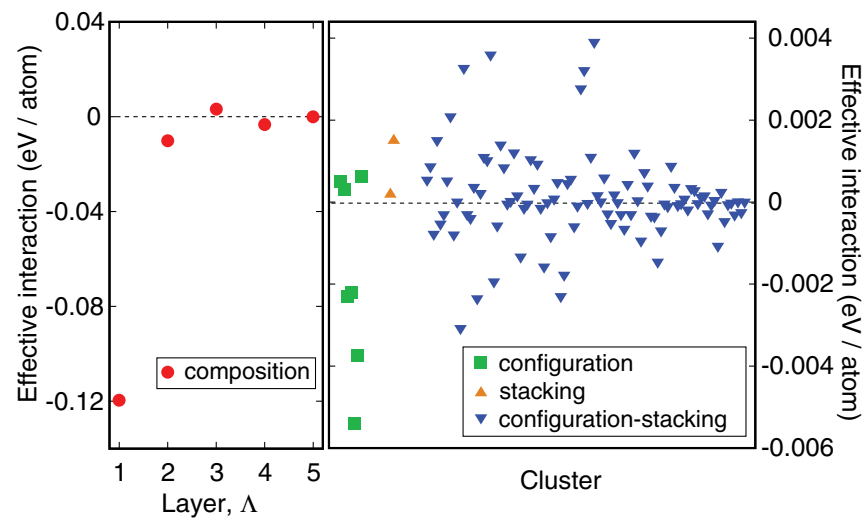

FIG. 2. (Color online) Contribution to the total energy at Pt-Re surface from composition (left) and atomic configuration, stacking, and coupling between configuration and stacking (right) in terms of Fig. 1. 


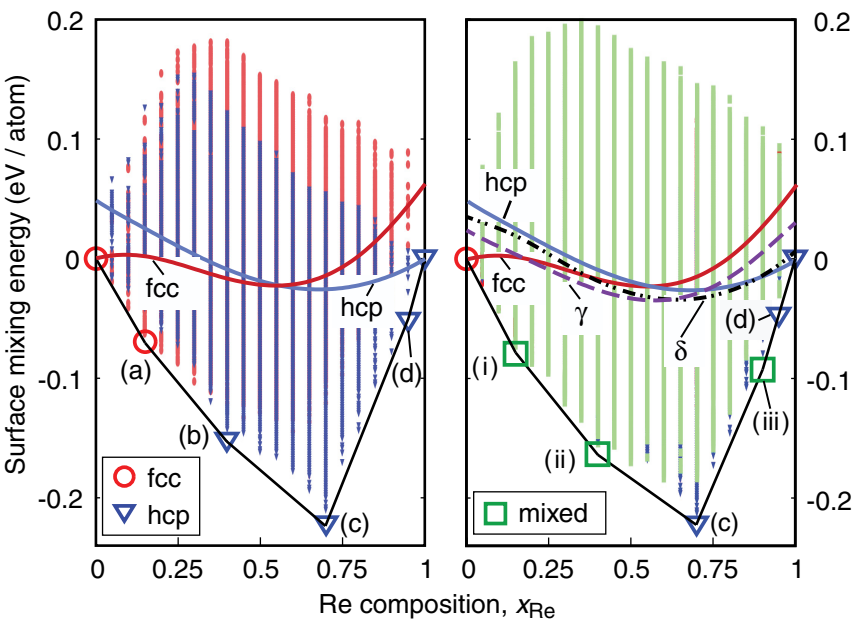

FIG. 3. (Color online) Surface mixing energy as a function of surface Re composition at the top five layers when fcc and hcp stackings are taken into account (left) and when all possible stacking sequences are taken into account (right). Mixing energy of ground-state surface structure with fcc, hcp, and mixed stackings is represented by large open circles, triangles, and squares, respectively, and that of other structures with fcc, hcp, and mixed stackings are represented by red, blue, and green lines. Ground states for the surface alloy are described by (a)-(d) and (i)-(iii). The solid and broken curves denote surface mixing energy for ideally random states with stackings that have lowest mixing energy for some composition range.

cannot be simply interpreted only by composition and atomic configurations, and coupling contributions should therefore play a significant role.

In order to assess which surface-ordered structure is formed under given surface composition, the surface ground-state diagram has been used powerfully ${ }^{13}$ where surface mixing energy for possible structures is described in terms of surface composition. We first show at the left-hand side of Fig. 3 the surface ground-state diagram of the Pt-Re alloy when only fcc- and hcp-stackings are considered. This is the "conventional" ground-state diagram that previous theoretical studies referred to, where stacking fault effects are neglected. Large open circles, triangles, and squares denote ground states with fcc, hcp, and mixed stacking, and vertical red, blue, and green lines denote other ordered structures with fcc, hcp, and mixed stacking, respectively. Solid and broken curves represent mixing energy for ideally random states with stackings that can be most stable at some composition range. The black lines connecting with the ground states denote the ground-state line, where surface with composition $x_{\mathrm{Re}}$ on the line energetically favor phase separation into two neighboring ground states. Note that surface mixing energy of ground states of (a)-(d) and (i)-(iii) are obtained by ECIs, which are also confirmed by DFT calculation within errors of the CV score, $0.1 \mathrm{meV} /$ atom. The diagram in Fig. 3 for ordered structures is obtained by MC simulation with up to 16 atoms at each layer with 11 layers based on simulated annealing algorism with gradually decreasing temperature from $10^{4}$ to $0 \mathrm{~K}$ in order to effectively sample possible atomic configurations, and that for random states is obtained by $\mathrm{MC}$ under canonical ensemble with 1024 atoms at each layer with 11 layers at
$10^{6} \mathrm{~K}$ where ideal random mixing at the surface is confirmed to be achieved. It can be clearly seen that between fcc Pt and hcp Re surfaces, there are four ground-state surface ordered structures of (a)-(d). The structure (a) has fcc stacking, while (b)-(d) have hcp stacking. Therefore, the surface with Pt-rich composition prefers fcc stacking while that with Re-rich composition prefers hcp stacking, which also holds for random states. This tendency has been reasonably found in the Pt-Re bulk phase diagram where the Pt-rich composition has an fcc structure while the Re-rich composition has hcp. ${ }^{35}$ The mixing energy for random states lies far above the ground-state lines, indicating the strong ordering tendency at the surface. The right-hand side of Fig. 3 is our "improved" surface ground-state diagram including stacking fault effects, i.e., including possible stacking sequences at the surface. We can clearly see that compared with the left-hand side of Fig. 3, two of four surface-ordered structures of (a) and (b) are no longer ground states, and (i) and (ii) with mixed stacking are ground states where their mixing energies are $\sim 0.01 \mathrm{eV} /$ atom lower than (a) and (b), respectively. No ground state in intermediate surface composition has fcc stacking. Moreover, there is an additional ground state at $x_{\mathrm{Re}}=0.9$ of (iii), which also has mixed stacking. For random states, an additional two mixed stackings have been found that have the lowest surface mixing energy for some composition ranges: One is $\gamma$ with $\mathrm{ACACBA} \cdots$, and another is $\delta$ with CABABA $\cdots$ stacking sequence, where the leftmost capital letter denotes the stacking sequence for the topmost layer. Note that in order to quantitatively describe surface phase stability at finite temperature, free energy calculation including contribution of configuration entropy and vibrational effects should be required. For ideally random states we consider, configuration entropy contribution should be the same for each stacking. Since again, we neglect vibrational contribution to the total energy, which can play a significant role at finite temperature, ${ }^{36}$ ground states in Fig. 3 are the most stable at $T=0 \mathrm{~K}$, while for random states, there is a possibility that stacking other than $\gamma$ or $\delta$ can be most stable due to the vibrational contributions. However, the present results of Fig. 3 certainly reveal that for the electronic contribution, the stacking fault significantly affects phase stability in random states to reverse stable stacking sequences. We summarize the structural details of the ground-state ordered structures in Table I, including surface composition, atomic configuration for the top three layers, interlayer distance between $r$ th and $s$ th layers, $d_{r s}$, and stacking sequences. It is clear that the topmost layer exhibits strong Pt segregation for the whole composition $x_{\mathrm{Re}}$, which is expected from the ECIs in Fig. 2, while at the second layer, Pt segregation depends on $x_{\mathrm{Re}}$, and the third layer tends to exhibit Pt depletion. Strong Pt segregation at the topmost layer as well as $\mathrm{Pt}$ depletion at the third layer has also been found by previous experiments of $\mathrm{Pt}_{25} \mathrm{Re}_{75}$ polycrystalline alloy. ${ }^{37} \mathrm{Pt}$ segregation at the surface has also been predicted for nanoparticles at $\mathrm{Pt}_{75} \mathrm{Re}_{25}$ in fcc configuration and at $\mathrm{Pt}_{25} \mathrm{Re}_{75}$ in hep configuration based on the embedded atom method. ${ }^{38}$ The interlayer distance between first and second layers, $d_{12}$, tends to be larger for the Pt-rich composition in the top two layers, which would be due mainly to the larger lattice constant of $\mathrm{Pt}$ than that of Re, while this does not hold for the interlayer distance of $d_{23}$. The three ground states of 
TABLE I. Atomic configuration, interlayer distance between $r$ th and $s$ th layers, $d_{r s}(\AA)$, and stacking sequence for ground-state surfaceordered structures at the bottom of Fig. 3. Bright circles denote Pt atoms, and dark circles denote Re atoms.

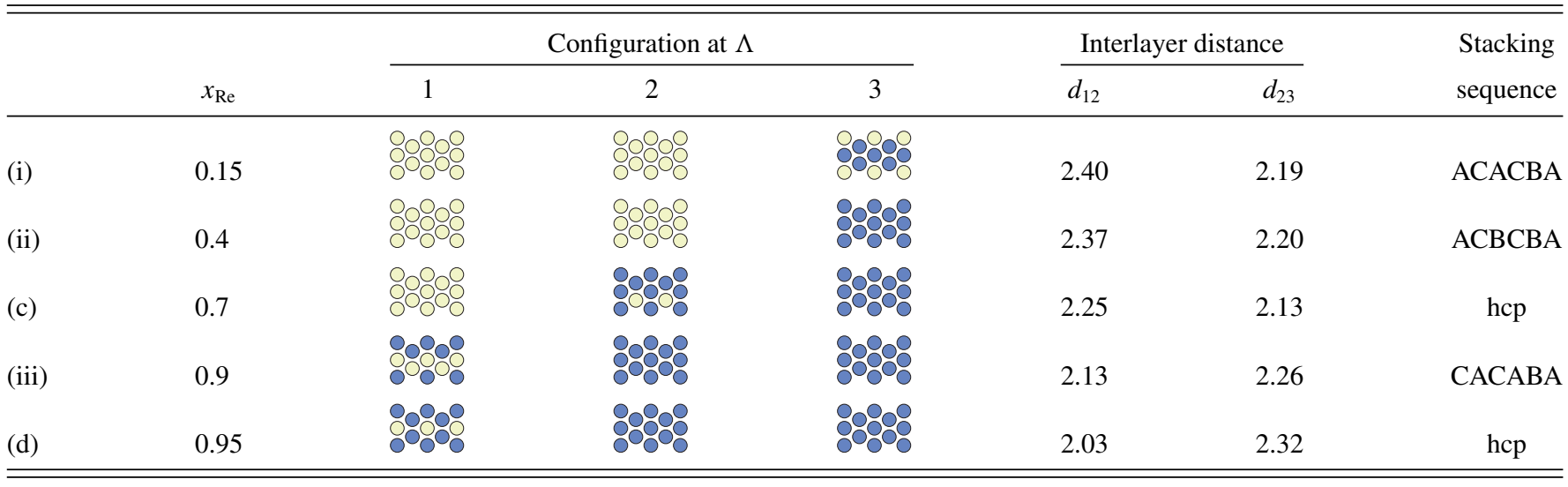

(i)-(iii) all have different stacking sequences, indicating that the stacking sequence for stable surface structures certainly depends on surface composition. This would be attributed to the significant coupling contributions between atomic configuration and stacking, shown in Fig. 2. Another important point is that random states have two mixed stackings of $\gamma$ and $\delta$ with the lowest mixing energy while no ground state has $\delta$ stacking [(i) has $\gamma$ stacking]. These findings certainly suggest that the consideration of stacking sequences only for surface ground states is not sufficient to describe surface phase stability for random states, and "full" consideration of possible stacking sequences is naturally required. The importance of the inclusion of stacking fault effects is particularly clear when we see the surfaces of (iii) and (d): While a difference in atomic configuration for the two surfaces is found only in the topmost layer, the stable stacking sequence differs due to the coupling between configuration and stacking.

It has also become important to assess surface electronic structures for ground states in order to see the inclusion of stacking fault effects. At $x_{\mathrm{Re}}=0.15$ and 0.4 , surface structures of (a), (i), (b), and (ii) have the same layer-confined atomic

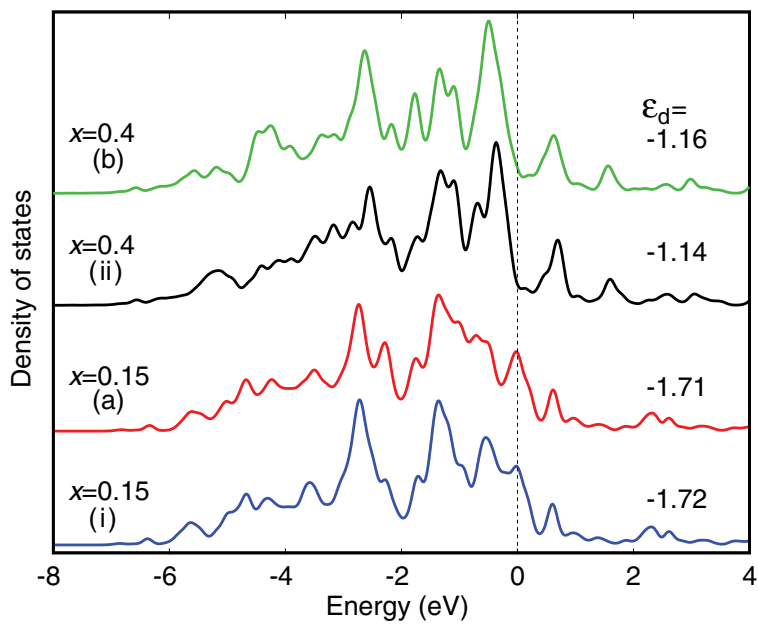

FIG. 4. (Color online) Electronic density of states at the top layer for Pt $d$ states of surface ground states of (i), (a), (ii), and (b) in Fig. 3. Fermi energy is set at zero. The $d$-band center measured from Fermi energy, $\epsilon_{\mathrm{d}}$, is described together. configurations at the top two layers (i.e., 100\% Pt). Therefore, we show in Fig. 4 the electronic density of states (DOS) at the top layer for $\mathrm{Pt} d$ states to see the electronic structure in terms of surface composition and stacking sequences. The center of gravity of the $d$ band, $\epsilon_{\mathrm{d}}$, is described together. It can be clearly seen that with the same surface composition, the characteristic shape of the DOS appears to be similar, and $\epsilon_{\mathrm{d}}$ is slightly different by $0.01-0.02 \mathrm{eV}$. Meanwhile, the shape and $\epsilon_{\mathrm{d}}$ exhibit a significant difference when surface composition of the top five layers, $x$, differs. This indicates that in the Pt-Re alloy, the stacking sequence is the predominant factor while the surface composition of underlying layers plays a significant role in the surface electronic structure. The slight difference in the surface electronic structure with the same surface composition is expected to be enhanced for systems with stronger coupling between stacking and composition or atomic configuration, which leads to different composition and stacking sequences in sublayers. In such system, knowing surface ground states, including stacking fault effects, would accelerate the design of thermodynamically stable surfaces with desirable electronic structures.

\section{CONCLUSION}

We assessed stacking fault effects on surface phase stability in alloys based on first-principles calculation with variablelattice cluster expansion (VLCE), enabling coupling effects between atomic configuration and stacking sequence on total energy to be modeled. As an example, the close-packed surface of fcc-hcp, Pt-Re alloys, was investigated. Our results show that there are several stable surface structures with stacking faults in ground states as well as random states. The stacking sequence for stable surface structures depends on surface composition, which is mainly due to the significant contribution of coupling effects. Our proposed formalism can successfully confirm the importance of stacking fault effects on surface phase stability in alloys.

\section{ACKNOWLEDGMENTS}

This study was supported by Toyota Physical \& Chemical Research Institute Scholars and Grant-in-Aid for Young Scientists B (Grant No. 22760502) from JSPS. 
${ }^{1}$ Y. Gauthier and R. Baudoing, Surface Segregation Phenomena, edited by P. A. Dowben and A. Miller (CRC, Boca Raton, FL, 1990).

${ }^{2}$ M. Guttmann and D. McLean, Interfacial Segregation, edited by W. C. Johnson and J. M. Blakely (American Society for Metals, Metals Park, OH, 1979).

${ }^{3}$ Y. G. Shen, D. J. O'Connor, K. Wandelt, and R. J. MacDonald, Surf. Sci. 328, 21 (1995).

${ }^{4}$ J. Y. Wang, J. du Plessis, J. J. Terblans, and G. N. van Wyk, Surf. Sci. 423, 12 (1999).

${ }^{5}$ H. Graupner, L. Hammer, K. Miiller, and D. M. Zehner, Surf. Sci. 322, 103 (1995).

${ }^{6}$ V. R. Stamenkovic, B. Fowler, B. S. Mun, G. Wang, P. N. Ross, C. A. Lucas, and N. M. Markovic, Science 315, 493 (2007).

${ }^{7}$ W. Chen, L. Severin, M. Gothelid, M. Hammar, S. Cameron, and J. Paul, Phys. Rev. B 50, 5620 (1994).

${ }^{8}$ J. Florencio, D. M. Ren, and T. T. Tsong, Surf. Sci. 345, L29 (1996).

${ }^{9}$ E. Platzgummer, M. Sporn, R. Koller, S. Forsthuber, M. Schmid, W. Hofer, and P. Varga, Surf. Sci. 419, 236 (1999).

${ }^{10}$ I. A. Abrikosov, A. V. Ruban, H. L. Skriver, and B. Johansson, Phys. Rev. B 50, 2039 (1994).

${ }^{11}$ A. V. Ruban and H. L. Skriver, Comput. Mater. Sci. 15, 119 (1999).

${ }^{12}$ R. Drautz, H. Reichert, M. Fahnle, H. Dosch, and J. M. Sanchez, Phys. Rev. Lett. 87, 236102 (2001).

${ }^{13}$ S. Müller, J. Phys.: Condens. Matter 15, R1429 (2003).

${ }^{14}$ L. V. Pourovskii, A. V. Ruban, B. Johansson, and I. A. Abrikosov, Phys. Rev. Lett. 90, 026105 (2003).

${ }^{15}$ O. Wieckhorst, S. Müller, L. Hammer, and K. Heinz, Phys. Rev. Lett. 92, 195503 (2004).

${ }^{16}$ B. C. Han, A. Van der Ven, G. Ceder, and B.-J. Hwang, Phys. Rev. B 72, 205409 (2005).
${ }^{17}$ W. G. Burgers, Physica 1, 561 (1934).

${ }^{18}$ Ch. Rath, J. E. Prieto, S. Müller, R. Miranda, and K. Heinz, Phys. Rev. B 55, 10791 (1997).

${ }^{19}$ Surface Alloys and Alloy Surfaces, edited by D. P. Woodruff (Elsevier, Amsterdam, 2002).

${ }^{20}$ K. Yuge, J. Phys.: Condens. Matter 22, 125402 (2010).

${ }^{21}$ K. Yuge, Phys. Rev. B 85, 144105 (2012).

${ }^{22}$ See Supplemental Material at http://link.aps.org/supplemental/ 10.1103/PhysRevB.87.024105 for the details of the 726 input structures used in DFT total-energy calculations.

${ }^{23}$ M. Stone, J. R. Stat. Soc. B 36, 111 (1974).

${ }^{24}$ D. M. Allen, Technometrics 16, 125 (1974).

${ }^{25}$ A. van de Walle, M. Asta, and G. Ceder, CALPHAD 26, 539 (2002).

${ }^{26}$ G. L. W. Hart, V. Blum, M. J. Walorski, and A. Zunger, Nat. Mater. 4, 391 (2005).

${ }^{27}$ A. van de Walle, Nat. Mater. 4, 362 (2005).

${ }^{28}$ K. Yuge, J. Phys.: Condens. Matter 22, 245401 (2010).

${ }^{29}$ K. Yuge, Phys. Rev. B 84, 134207 (2011).

${ }^{30}$ G. Kresse and J. Hafner, Phys. Rev. B 47, R558 (1993).

${ }^{31}$ G. Kresse and J. Furthmüller, Phys. Rev. B 54, 11169 (1996).

${ }^{32}$ G. Kresse and D. Joubert, Phys. Rev. B 59, 1758 (1999).

${ }^{33}$ P. E. Blöchl, Phys. Rev. B 50, 17953 (1994).

${ }^{34}$ J. P. Perdew, K. Burke, and M. Ernzerhof, Phys. Rev. Lett. 77, 3865 (1996).

${ }^{35}$ T. B. Massalski, Binary Alloy Phase Diagrams, 2nd ed. (ASM Intl., Materials Park, OH, 1990).

${ }^{36}$ A. van de Walle and G. Ceder, Rev. Mod. Phys. 74, 11 (2002).

${ }^{37}$ B. S. Mun, M. Rossi, and P. N. Ross Jr., J. Chem. Phys. 129, 174707 (2008).

${ }^{38}$ G. Wang, M. A. van Hove, P. N. Ross, and M. I. Baskes, J. Chem. Phys. 121, 5410 (2004). 